\title{
Can Artificial Intelligence Change the Way in Which Companies Recruit, Train, Develop and Manage Human Resources in Workplace?
}

\author{
Faiyaz Md. Iqbal ${ }^{1}$
}

${ }^{`} \mathrm{C} / \mathrm{O}$ Advocate Farid Uddin Akhtar, 61/1 Naya Paltan (3rd floor), Opposite Paltan Thana, Dhaka-1000, Bangladesh

Email:smtak_faiyaz@yahoo.com Tel: +8801928762091

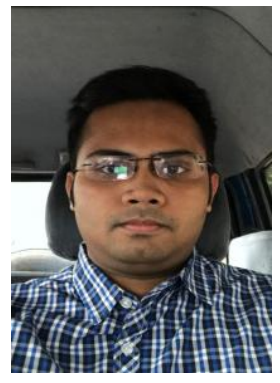

\begin{abstract}
The purpose of this research is to find out the impact that Artificial Intelligence (AI) is having on the recruitment, training, development and retention of workers in organizations. Although, a very recent phenomena in the Human Resource (HR) world, AI is already having significant impact in this sector and this paper focuses on those impacts in details. The research focuses on some external articles to demonstrate some of the findings of the impact of AI in modern day organizations. The study finds that AI is having positive impacts on the management of workers in companies and comes up with a recommendation to invest more in AI processes to take HR management to the next level.
\end{abstract}

Keywords: Artificial intelligence, Human resource management, Revolutionizing recruitment, Training and development of human resources, Preventing employee turnover.

Citation | Faiyaz Md. Iqbal (2018). Can Artificial Intelligence Change the Way in Which Companies Recruit, Train, Develop and Manage Human Resources in Workplace? Asian Journal of Social Sciences and Management Studies, 5(3): 102-104. History:

Received: 25 April 2018

Revised: 23 May 2018

Accepted: 28 May 2018

Published: 31 May 2018

Licensed: This work is licensed under a Creative Commons

Attribution 3.0 License (oc) E E

Publisher: Asian Online Journal Publishing Group
Funding: This study received no specific financial support.

Competing Interests: The author declares that there are no conflicts of interests regarding the publication of this paper.

Transparency: The author confirms that the manuscript is an honest, accurate, and transparent account of the study was reported; that no vital features of the study have been omitted; and that any discrepancies from the features of the study have been omitt
study as planned have been explained.

study as planned have been explained.
Ethical: This study follows all ethical practices during writing.

\section{Contents}

1. Introduction

2. Methodology of the Paper ..........103

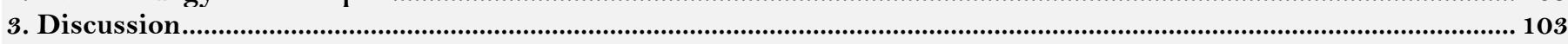

4. Conclusion .

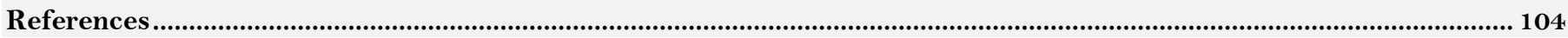




\section{Introduction}

Artificial Intelligence has had a major impact in transforming the way in which companies have traditionally recruited, trained and developed people in organizations. Moreover, predictive and other AI technology has also made it possible for HR managers to target and attract staffs who would otherwise leave the organization, reducing the cost and hassles associated with employee turnover. The research analyzes in details how AI is revolutionizing all these aforementioned aspects of managing people in organizations. It also goes on to explore some of the disadvantages of AI processes in HR and comes up with suggestions to incorporate some form of human control in the AI-aided HR processes to counter the deficiencies. It acknowledges the growing importance and likewise increasing use of AI in the HR world and encourages more investment in the AI processes to make them more efficient.

\section{Methodology of the Paper}

The research paper uses valuable inputs from a number of other Articles mentioned in the references section to illustrate how Artificial Intelligence is becoming an increasingly significant tool used by modern day HR managers in organizations to source, recruit, train, develop as well as retain the best possible employees. The input obtained from the Articles help to discuss in details the type of AI-processes currently in place to source the best possible employees and facilitate recruitment. It also points to the impact of AI-aided technology in training and developing potential among employees as well as taking measures to retain talented staffs. In addition the cases of UnderArmour and FirstJob are discussed as aid to vividly illustrating how AI-aided systems are simplifying the recruitment processes, again with the aim of showing the enormous contribution AI-aided processes are making in the HR world.

\section{Discussion}

Artificial Intelligence (AI) is a very recent phenomena in the Human Resource (HR) world, making it's mark felt in the HR world as recent as 2017. Nonetheless it has made a significant impact in people management, making it's significance felt in the recruitment, training, development and retention of employees in the workplace (SextonBrown, 2018). According to Human Capital Trends Report by Deloitte, $38 \%$ of companies in US already use AI methods in HR management while $62 \%$ expect to do so by the end of 2018 (Erickson, 2018). That being said, just like with any change, the transformation in adopting Artificial Intelligence in Human Resource is fraught with some drawbacks and uncertainties. However, this new trend towards making HR practices dependent on Machine Learning and AI tools has the potential of leading to massive gains in terms of enhancing employee motivation and productivity in the workplace.

Artificial Intelligence has revolutionized the way in which companies recruit employees. Unfortunately, after 60 years of traditional recruiting, where employers pay placement fees and almost 15 years since LinkedIn was founded, nothing dramatic changed in the global recruitment industry prior to the arrival of Artificial Intelligence in HR. Relying on LinkedIn and other traditional methods to attract and recruit the right employees has not been successful in recent times since most companies these days want passive candidates- those who already have jobs and are not seeking to make a move. Switching to adopting Artificial Intelligence can help companies find these passive talents. That is because AI uses bots to crawl the web and scour hundreds of sites including personal websites, meetup groups and tech chat rooms, in addition to traditional social media sites. The bots not only find a good match for the job but they also predict the likelihood that someone is open to a job change, making it efficient for companies to poach talents which are rare to find (Almog, 2018).

Moreover, by using AI-aided technology, companies can make the recruitment process much quicker and smoother. For instance, Under Armour, an American company which manufactures footwear, sports and casual apparel receives more than 30,000 resumes per month. Handling so many resumes from prospective candidates is indeed a very difficult task and unsurprisingly, the company's recruitment process was cumbersome, where it was difficult to monitor or track applicants properly. To solve this problem, the company put in place an AI-enabled digital recruitment system called Hirevue to bring innovation to their recruitment process. Using this system, managers in the organization could create candidate interview with pre-recorded questions. Only the candidates who met the requirements were then invited for interview via webcam or mobile devices, enabling retail managers to hire the best candidates very quickly. Another instance of a company using AI-aided technology to improve recruitment efficiency is illustrated by FirstJob, an online recruitment firm implementing Mya. It is an AI recruiting assistant which can talk to thousands of candidates at once. It asks candidates pre-screening questions, ranks them based on pre-defined factors and alerts them when a position has been filled. By using this AI-aided technology, hiring managers at FirstJob do not have to spend time managing large candidate pool and instead can only focus on interviews and closing offers. Thus AI technology can change the process of candidate sourcing and recruiting them from manual to automated processes, saving time and money for hiring managers. In addition, AI algorithms can cure the problem of poor job descriptions, which a company's poorly drafted exhaustive list of job requirements may make. AI algorithms can apply different skills scenarios, allowing employers to see how minor revisions in the description of jobs can return different results in real time. This can help employers move away from the habit of publishing unrealistic exhaustive job requirements to advertising descriptions which reflect what is truly essential for the job, helping to find the right fit.

Nevertheless, while the Artificial Intelligence technology can render remarkable results, the human element in hiring is also important. Recruiting managers need to work with technology leaders in their business to assess which Artificial Intelligence tools and processes work best, to ensure that the results derived from using AI in recruiting can help in finding and attracting the best talents (Almog, 2018).

Artificial Intelligence is already starting to have a huge impact in the way in which companies train and develop their employees. When recruiting and inducting new employees, the main challenge is to rapidly, yet efficiently give them a thorough knowledge and understanding of the organization's internal policies and procedures. Interestingly, the prospect of adding machine-learning capabilities to various HR software systems to facilitate with induction training has arrived (Baillie-Stewart, 2017). According to Ari Kopoulos, writer for 
EmployeeConnect.com, AI can handle many of the employees' routine requests about the organization's policies and benefits. Moreover, machine learning AI can recommend training programs and external events that would interest an employee for better holistic management. Machine learning AI is also providing many vocational training to employees, thus freeing up considerable time for HR managers to focus on other priorities.

Mass personalization of information, especially around employee training and development can have a significant effect on the HR functions of organizations. The good news is that very recently, AI-enriched learning systems are able to offer customizable, employee related training programs based on individual employee performances. These are likely to facilitate enormous increases in workforce productivity as these AI-learning systems can act as personal tutors to employees, helping to develop their potential and capability (Baillie-Stewart, 2017).

Nonetheless using Artificial Intelligence in training and development of employees carries some disadvantages as well. AI processes can take over repetitive tasks formerly performed by humans in organization leading to redundancy for these positions. Nonetheless, these drawbacks can be nullified by using AI processes to improve the digital skills of employees, in which case their former repetitive jobs become boring to them in any event and employees replaced by AI in repetitive jobs can now perform digitally enriched jobs after undergoing training with the use of these AI processes. Moreover, constant human control and intervention can be used to minimize the risks associated with transitioning to AI systems in an organization.

AI can also be used to understand how employees are doing within an organization and at times taking right steps to prevent turnover. Employee turnover is a significant area, which if better handled, can save a lot of money for the organizations. Companies can use AI assisted predictive technology to reduce employee turnover. Such technology can be used to determine when an employee's engagement falls. This data can then be used by HR managers to proactively intervene and provide vital support to help solve the problems the employee is facing within the organization. This in turn will lead to lower turnover and save costs associated with the replacement of human capital (Fraser, 2018). AI technology can also help companies to spot patterns in historic data to predict future behavior. If the data shows that staff engagement falls at a certain point for specific roles or upon attaining certain milestones, companies can use that data to take steps to break that pattern recurring in the future. If the AI obtained data leads to a more profound revelation, for example, that the company acts as a stepping stone for millennials in their careers, then being mindful of this pattern can help ensure that recruitment is set up to deal with this engagement curve (Fraser, 2018). This helps to ensure that customers of the organization do not suffer due to unpredictable employee churn.

\section{Conclusion}

Artificial Intelligence has already made significant contributions in the HR world through revolutionizing the way that recruitment, training and development takes place in organizations. It has made it possible for companies to advertise themselves to attract the best possible talents, even if that means attracting passive candidates, something which traditional recruitment processes and LinkedIn failed to offer. Moreover, AI algorithms have made it possible for recruiters to see in real time how revising job descriptions to reflect what is truly essential can help attract and find the best possible talents. Moreover, AI enriched machine learning is providing employees with induction training and the support of other vocational courses to acclimatize to workplace environment. What is more, mass personalization of information is now possible with AI-enriched learning systems that are able to provide employees with customizable training programs that better address their needs and priorities. AI enriched systems have also been able to reduce employee turnover in organizations by analyzing data obtained from AIassisted predictive and other technologies and taking preventive steps to reduce staff turnover. However, there is always a need for human intervention to determine which AI-assisted technologies work best to render highest workforce productivity increases and to counter the effects of AI-technology replacing many repetitive manual jobs. Nevertheless, AI-technology should be seen as a key area for investment by HR managers as the technology has the potential to take Human Resource Management to the next level.

\section{References}

Almog, G., 2018. Traditional recruiting isn't enough: How ai is changing the rules in the human capital market. Baillie-Stewart, A., 2017. Artificial intelligence for employee training and development.

Erickson, R., 2018. Infographic: Insights into a highly mature talent acquisition team. HR Daily Advisor.

Fraser, S., 2018. Preventing employee turnover with artificial intelligence.

Sexton-Brown, E., 2018. How is artificial intelligence developing HR? HRD Connect. 